\title{
DIVERSIDAD GENÉTICA EN DOS HUERTOS SEMILLEROS DE Pinus patula SCHIEDE ex SCHLTDL. et CHAM.
}

\author{
GENETIC DIVERSITY AT TWO SEED ORCHARDS \\ OF Pinus patula SCHIEDE ex SCHLTDL. et CHAM.
}

\section{Mónica Méndez-Neri ${ }^{1}$ Carlos Ramírez-Herrera ${ }^{1}$ *, J. Jesús Vargas-Hernández ${ }^{1}$, Tomás Martínez-Trinidad', Javier López-Upton ${ }^{1}$ y Pedro Antonio López ${ }^{2}$}

\begin{abstract}
${ }^{1}$ Colegio de Postgraduados, Campus Montecillo, Posgrado en Ciencias Forestales, Montecillo, Texcoco, Estado de México, México. ${ }^{2}$ Colegio de Postgraduados, Campus Puebla, Cholula, Puebla, México.
\end{abstract}

\section{RESUMEN}

\begin{abstract}
La diversidad genética es importante en los huertos semilleros para producir plantas con alta capacidad de adaptación a cambios ambientales; por lo tanto, el objetivo del presente estudio fue evaluar la diversidad y estructura genética de dos huertos semilleros asexuales de Pinus patula y determinar el grado de diferenciación de cada huerto. El primer huerto se localiza en el ejido La Selva, municipio de Huayacocotla, Veracruz, México y el segundo en el ejido Villa Cuauhtémoc, municipio de Chignahuapan, Puebla, México. Cada huerto se estableció con árboles clonados fenotípicamente superiores seleccionados en su propia área. Muestras de follaje se recolectaron de un rameto de cada clon en ambos huertos semilleros. Los marcadores moleculares de microsatélite NZPR1078, NZPR114, NZPR5, PtTX2123, PtTX2146, PtTX3107 y PtTX3127 se usaron para realizar el análisis de diversidad genética. El número promedio de alelos por locus fue 9.9 para ambos huertos semilleros. La heterocigosidad observada y la esperada fueron 0.602 y 0.693 , respectivamente. El índice de fijación fue 0.125 . Un déficit de heterocigotos se encontró cuando los dos huertos semilleros se analizaron en conjunto $\left(F_{\mathrm{IT}}=0.125\right)$ y dentro de cada uno de estos $\left(F_{I S}=0.105\right)$. El índice de diferenciación fue bajo $\left(F_{S T}=0.023\right)$. La mayor parte de la diversidad genética se encontró dentro de los huertos con sólo $4.5 \%$ entre éstos. La diversidad genética fue alta en los huertos asexuales de $P$. patula con deficiencias moderadas de heterocigotos y un nivel bajo de diferenciación entre ambos huertos semilleros.
\end{abstract}

Palabras clave: Estructura genética, heterocigosidad, índice de fijación, microsatélites.

\section{SUMMARY}

Genetic diversity is important in seed orchards to produce plants with high capability of adaptation to environmental changes; thus, the objective of this study was to evaluate the genetic diversity and structure of two asexual seed orchards of Pinus patula and to determine the degree of differentiation of each orchard. The first orchard is located at common land La Selva, municipality of Huayacocotla, Veracruz, Mexico and the second one at common land Villa Cuauhtémoc, municipality of Chignahuapan, Puebla. Mexico. Each orchard was established with phenotypically superior cloned trees selected in their own area. Foliage samples were collected from one ramet from each clone in both orchards. Microsatellite molecular markers NZPR114, NZPR5, PtTX2123, PtTX2146, PtTX3107 and PtTX3127 were used to perform the genetic diversity analysis. The average number of alleles per locus was 9.9 for both seed orchards. The observed and expected heterozygosities were 0.602 and 0.693 , respectively. The fixation index was 0.125 . A deficit of heterozygotes was found when both seed orchards were analyzed jointly $\left(F_{\text {IT }}=125\right)$ and within each of the orchards $\left(F_{1 S}=105\right)$. The differentiation index was low $\left(F_{S T}=0.023\right)$. Most of the genetic diversity was found within the orchards with only $4.5 \%$ between them. The genetic diversity was high in the asexual orchards of $P$. patula with moderate deficiency of heterozygotes and low level of differentiation between both seed orchards.

Index words: Fixation index, genetic structure, heterozygosity, microsatellites.

\section{INTRODUCCIÓN}

Pinus patula Schiede ex Schltdl. et Cham. es una especie monoica y anemófila endémica de México (Perry, 1991) que se considera importante para el establecimiento de plantaciones comerciales en zonas tropicales y subtropicales con fines de producción de madera (Dvorak et al., 2000). Los programas de mejoramiento genético de $P$. patula se iniciaron en Sudáfrica en la década de los 1970 s (Dvorak et al., 2000). En México, los primeros esfuerzos de selección fenotípica y evaluación genética para incrementar productividad y calidad de la madera de P. patula se iniciaron en la década de los 1990s (Sáenz-Romero et al., 1994; Valencia et al., 1995). Dada la importancia y considerando las dificultades prácticas del abastecimiento de semilla para reforestación (Ortiz-Bibian et al., 2019), en 2012, la Comisión Nacional Forestal (CONAFOR) financió el establecimiento de tres huertos semilleros asexuales de esta especie con el fin de satisfacer la demanda de semilla para la reforestación de áreas bajo manejo forestal.

La diversidad genética es esencial para que los grupos de individuos de una especie se adapten a cambios ambientales (Frankham et al., 2004); también, ésta es importante para la selección de individuos que poseen características sobresalientes de interés económico, como tamaño y forma de tronco, velocidad de crecimiento, calidad de la madera y resistencia a enfermedades o

Recibido: 1 de abril de 2019

Aceptado: 15 de enero de 2020 
a factores ambientales extremos (White et al., 2007). En una especie, la diversidad genética es producto de diferencias genéticas entre individuos en una población y entre poblaciones (Nei, 1977). Las diferencias genéticas entre poblaciones se deben al reducido o nulo intercambio de migrantes como consecuencia del aislamiento por barreras o distancia entre poblaciones de una especie (Wright, 1969). El aislamiento de poblaciones promueve el apareamiento entre individuos emparentados, lo que aumenta la probabilidad de que alelos diferentes se fijen y conduzcan a una diferenciación entre poblaciones (Wright, 1969). Esta partición de la diversidad genética se conoce como estructura genética, la cual es importante en el establecimiento de las unidades de manejo y conservación de una especie (Allendorf y Luikart, 2007). Un huerto semillero es una unidad de manejo de una especie.

Un huerto semillero es una plantación donde individuos sobresalientes crecen y se cruzan entre sí en una localidad para producir germoplasma de calidad genética superior para su uso en los programas de plantaciones forestales (White et al., 2007). La meta del establecimiento de un huerto semillero es maximizar las ganancias genéticas en características de interés para conservar un nivel alto de diversidad genética al seleccionar árboles o clones no emparentados (Ahlinder et al., 2014); sin embargo, la propagación vegetativa disminuye la diversidad genética lo que puede limitar su uso en la silvicultura (Stelzer y Goldfarb, 1997), pero ésta es de amplio uso en el establecimiento de plantaciones comerciales para la propagación de genotipos genéticamente superiores (White et al., 2007).

Los marcadores moleculares son una herramienta útil para estimar la diversidad genética, estructura genética, flujo génico y sistemas de apareamiento en poblaciones de cruzamiento de especies del género Pinus (Çengel et al., 2012). Los microsatélites son marcadores que se utilizan ampliamente para medir la diversidad y estructura genética en especies forestales (Barrios et al., 2004; Gaspar et al., 2009; Zelener et al., 2005).

La diversidad y estructura genética se han cuantificado a través de marcadores aloenzimáticos en poblaciones naturales de P. patula (Ramírez-Herrera et al., 1998), mediante ISSRs en $P$. cembroides (Fuentes-Amaro et al., 2019) y de microsatélites en P. patula (Alfonso-Corrado et al., 2014) y en P. oocarpa (Dvorak et al., 2009). La mayoría de las especies del género Pinus tienen una diversidad genética alta y una diferenciación baja entre poblaciones (Chhatre y Rajora, 2014; Chudzińska et al., 2016; Dvorak et al., 2009).

En los huertos semilleros es deseable una diversidad alta para continuar con la selección de individuos sobresalientes en generaciones avanzadas y mantener individuos con crecimiento rápido y posibilidad de adaptación a los cambios ambientales. La presente investigación se realizó con el objetivo de evaluar la diversidad y estructura genética de dos huertos semilleros asexuales de P. patula, bajo la hipótesis de que la diversidad genética es alta en estos huertos debido a que los clones se originaron de ortetos no emparentados mientras que la diferenciación genética es baja.

\section{MATERIALES Y MÉTODOS}

\section{Áreas de estudio}

Los hueros semilleros de Pinus patula var. patula se establecieron con apoyo de la Comisión Nacional Forestal (CONAFOR) en 2012. El primer huerto semillero, con 83 clones, se localiza en el ejido La Selva, municipio de Huayacocotla, Veracruz, México en las coordenadas geográficas $20^{\circ} 35^{\prime} 22^{\prime \prime} \mathrm{N}, 98^{\circ} 31^{\prime} 00^{\prime \prime} \mathrm{O}$ a una altitud de $1875 \mathrm{~m}$, mientras que el segundo, con 77 clones, se ubica en el ejido de Villa Cuauhtémoc, municipio de Chignahuapan, Puebla, México en las coordenadas geográficas $19^{\circ} 45^{\prime}$ $58^{\prime \prime} \mathrm{N}, 98^{\circ} 06^{\prime} 40^{\prime \prime} \mathrm{O}$ a una altitud de $2626 \mathrm{~m}$. Los ortetos (árboles donde se obtuvieron las yemas para producir los rametos en los huertos) se seleccionaron en el área forestal propiedad de cada uno de los ejidos.

\section{Toma de muestras}

En marzo de 2016 se recolectó follaje joven de un rameto de cada uno de los 160 clones de los huertos. El follaje se colocó en bolsas de plástico marcadas con el número del clon. Una muestra de $100 \mathrm{mg}$ de follaje fresco de cada clon se utilizó para la extracción del ADN según el protocolo ChargesSwitch ${ }^{\circledR}$ gDNAPlant Kit (Qiagen, Chatsworth, California, EUA). Se probaron 11 iniciadores de microsatélites, de los cuales amplificaron tres (NZPR5, NZPR114 y NZPR1078) desarrollados para Pinus radiata D Don (Chagné et al., 2004; Fisher et al., 1998) y cuatro (PtTX2123, PtTX2146, PtTX3107 y PtTX3127) para Pinus taeda L. (Elsik et al., 2000).

La reacción de amplificación por PCR se realizó en un volumen de reacción de $25 \mu \mathrm{L}$ con 20 ng de ADN genómico, $1 \mathrm{pmol}$ del iniciador hacia adelante, $1 \mathrm{pmol}$ del iniciador en reversa, $0.5 \mathrm{mM}$ de dNTPs, 5 X de Buffer Taq, $2 \mathrm{mM}$ de $\mathrm{MgCl}_{2}$ y 0.4 unidades de ADN Taq polimerasa. La amplificación se hizo en un Termociclador GeneAmp $® P C R$ System 9700 (Applied Biosystems, Foster City, California, EUA). La PCR se completó con el protocolo siguiente: 15 min a 95 ${ }^{\circ} \mathrm{C}$; 30 ciclos de $30 \mathrm{~s}$ a $94^{\circ} \mathrm{C}$ (desnaturalización); $60 \mathrm{~s}$ a $60.9^{\circ} \mathrm{C}$ (NZPR5), $64.3^{\circ} \mathrm{C}$ (NZPR1078), $59.1^{\circ} \mathrm{C}$ (PtTX2146, PtTX3127 y NZPR114) y $58.3^{\circ} \mathrm{C}($ PtTX2123 y PtTX3107) 
(alineamiento) y 1 min a $72{ }^{\circ} \mathrm{C}$ (extensión); todos seguidos por 15 min de extensión final a $72^{\circ} \mathrm{C}$. Los productos que resultaron de la amplificación de la PCR se separaron en un secuenciador automático Genetic Analyzer (AB 3130® Applied Biosystems) y estos se analizaron con el programa GeneMapper® V. 4.0 (Applied Biosystems).

\section{Estimación de parámetros y análisis de la información}

El número promedio de alelos por locus (A), la heterocigosidad observada $(\mathrm{Ho})$ y la esperada $(\mathrm{He})$ y el índice de fijación (F) se estimaron para cada uno de los huertos semilleros con Excel 2013 Microsoft Office®. La estructura genética se analizó utilizando los estadísticos $\mathrm{F}$ de Wright (Wright, 1965). También se realizó un análisis de varianza molecular con el programa GenAlEx6.5 (Peakall y Smouse, 2012).

\section{RESULTADOS}

\section{Diversidad genética}

Sesenta y nueve alelos se encontraron en los siete loci que se analizaron para P. patula en el presente estudio. El número de alelos por locus varió entre cinco y 15 para los loci PtTX2123 y PtTX2146, respectivamente. Cada uno de los loci PtTX3107, NZPR5, PtTX3127 y PtTX2146 tuvieron un alelo privado en el huerto semillero Villa Cuauhtémoc, mientras que los siete loci que se incluyeron en el presente estudio presentaron al menos un alelo privado en el huerto semillero en La Selva. Los loci NZPR5 y PtTX2146 tuvieron cada uno cuatro alelos privados en el huerto semillero de La Selva.

El número promedio de alelos por locus fue mayor en el huerto semillero La Selva (Cuadro 1); sin embargo, la heterocigosidad observada fue $18.7 \%$ superior en el huerto de Villa Cuauhtémoc con respecto al del huerto de La Selva. La heterocigosidad esperada también fue mayor en $3.5 \%$ en el huerto semillero Villa Cuauhtémoc. Por su parte, el índice de fijación fue mayor en el huerto semillero La Selva.

\section{Estructura genética}

Los valores totales del índice de fijación total $\left(F_{\mid T}\right)$ e índice de fijación dentro de poblaciones $\left(F_{I S}\right)$ fueron positivos (Cuadro 2). Los valores de $F_{I T}$ y $F_{I S}$ fueron positivos para los loci NZPR1078, NZPR1 14, NZPR5 y PtTX3107, y negativos para los loci PtTX2123, PtTX2146 y PtTX3127. Los loci NZPR5 y NZPR1078 tuvieron los valores mayores de $F_{I T}$ mientras que el locus PtTX2123 mostró el menor valor El locus NZPR1078 tuvo el mayor valor de $F_{\text {IS }}$. El valor del índice de fijación entre huertos semilleros o índice de diferenciación $\left(F_{S T}\right)$ fue bajo. El mayor valor de $F_{S T}$ fue para el locus NZPR5, mientras que el valor menor se encontró en el locus PtTX2123. Con base en el análisis de varianza molecular, la mayor diversidad genética se encontró dentro de árboles mientras que la menor diversidad genética se encontró entre los huertos semilleros (Cuadro 3).

\section{DISCUSIÓN}

La diversidad genética fue alta en ambos huertos semilleros de $P$. patula con base en el número total de alelos, el número promedio de alelos por locus, la heterocigosidad observada y la esperada. Una diversidad genética alta en los huertos semilleros es deseable para tener mayor posibilidad de que la descendencia se adapte a cambios ambientales y evitar depresión endogámica en generaciones avanzadas de selección dentro de programas de mejoramiento genético (Hartl y Clark, 2007) Por lo anterior, es deseable un número alto de clones genéticamente diferentes en los huertos semilleros a fin de mantener un nivel alto de diversidad genética y poder continuar con la selección de árboles con altas tasas de supervivencia y crecimiento, y tolerantes a plagas y enfermedades, sin disminuir la capacidad de adaptación a cambios ambientales.

El número total de alelos es un reflejo de alta diversidad en los huertos semilleros asexuales de P. patula en los Ejidos de la Selva y de Villa Cuauhtémoc. El número total de alelos en esta especie fue superior al número de alelos que se encontró en un huerto semillero constituido por 61 clones de Pinus koraiensis Siebold \& Zucc., con un total de

Cuadro 1. Parámetros de diversidad genética en dos huertos semilleros de Pinus patula.

\begin{tabular}{lcccc}
\hline Huerto semillero & $\begin{array}{c}\text { Número de alelos por } \\
\text { locus }\end{array}$ & $\begin{array}{c}\text { Heterocigosidad } \\
\text { observada }\end{array}$ & $\begin{array}{c}\text { Heterocigosidad } \\
\text { esperada }\end{array}$ & Índice de fijación (F) \\
\hline Villa Cuauhtémoc & $7.7 \pm 1.1$ & $0.652 \pm 0.09$ & $0.684 \pm 0.05$ & $0.047 \pm 0.13$ \\
La Selva & $9.3 \pm 1.2$ & $0.549 \pm 0.07$ & $0.660 \pm 0.08$ & $0.169 \pm 0.08$ \\
Ambos huertos & $9.9 \pm 1.2$ & $0.602 \pm 0.06$ & $0.688 \pm 0.06$ & $0.125 \pm 0.10$ \\
\hline
\end{tabular}


Cuadro 2. Valores de los estadísticos F de Wright para siete loci polimórficos en dos huertos semilleros asexuales de Pinus patula.

\begin{tabular}{lccc}
\hline Locus & Índice de fijación total $\left(F_{\text {IT }}\right)$ & $\begin{array}{c}\text { Índice de fijación } \\
\text { dentro de los huertos }\left(F_{\text {IS }}\right)\end{array}$ & $\begin{array}{c}\text { Índice de fijación } \\
\text { entre huertos }\left(F_{S T}\right)\end{array}$ \\
\hline NZPR1078 & 0.371 & 0.356 & 0.024 \\
NZPR114 & 0.112 & 0.102 & 0.011 \\
NZPR5 & 0.372 & 0.334 & 0.057 \\
PtTX2123 & -0.292 & -0.349 & 0.042 \\
PtTX2146 & -0.006 & -0.011 & 0.005 \\
PtTX3107 & 0.305 & 0.302 & 0.003 \\
PtTX3127 & -0.174 & -0.223 & 0.040 \\
Total & 0.125 & 0.105 & 0.023 \\
\hline
\end{tabular}

Cuadro 3. Análisis de varianza molecular de diversidad genética de Pinus patula.

\begin{tabular}{lccccc}
\hline Fuente & $\begin{array}{c}\text { Grados de } \\
\text { libertad }\end{array}$ & $\begin{array}{c}\text { Suma de } \\
\text { cuadrados }\end{array}$ & $\begin{array}{c}\text { Cuadrados } \\
\text { medios }\end{array}$ & $\begin{array}{c}\text { Estimación de } \\
\text { varianza }\end{array}$ & $\begin{array}{c}\text { Porcentaje (\%) } \\
\text { de varianza }\end{array}$ \\
\hline Entre huertos & 1 & 21.330 & 21.330 & 0.115 & 4.5 \\
Entre individuos & 158 & 472.707 & 2.992 & 0.557 & 21.8 \\
Dentro de huertos & 160 & 300.500 & 1.878 & 1.878 & 73.7 \\
Total & 319 & 794.538 & & 2.550 & 100 \\
\hline
\end{tabular}

56 alelos en 13 loci (Feng et al., 2010); además, el resultado aquí obtenido fue mayor al número total (45) de alelos de cinco microsatélites que se reportaron en Pinus resinosa Ait. (Boys et al., 2005); sin embargo, el número total de alelos que se reportaron para Pinus oocarpa Schiede ex Schlechtendal, Pinus tecunumanii (Schw.) Eguiluz et Perry, y P. patula (Dvorak et al., 2009) fue menor que el valor para los mismos siete loci del presente estudio (136). La diferencia en el número total de alelos se puede deber al número y distribución de las poblaciones incluidas en el muestreo de ambos estudios. Los clones en los huertos semilleros sólo provinieron de dos poblaciones de $P$. patula, mientras que Dvorak et al. (2009) muestrearon tres diferentes especies con un número mayor de poblaciones (51 poblaciones de P. oocarpa, 11 de P. tecunumanii y seis de P. patula) que se localizan en la mayor parte del área de distribución natural de estas especies. El número total de alelos también fue menor que el número que se encontró (105 alelos) en 10 microsatélites de 24 clones de P. radiata (Devey et al., 2002).

El número encontrado de alelos por locus (A) es muestra de una diversidad genética alta en $P$. patula var. patula en el presente estudio. Este estimador de diversidad genética fue mayor que el número promedio de alelos por locus $(A=8.5)$ que se encontró en 11 loci en cinco poblaciones naturales de $P$. patula var. patula y muy superior al valor (A $=4.55)$ que se encontró en una población de P. patula var. longipeduculata (Dvorak et al., 2009). La diferencia en el número de alelos promedio en ambos estudios se puede deber a que diferentes individuos en diferentes poblaciones se incluyeron en cada estudio, por lo que ambos estudios para la misma especie se pueden considerar complementarios; también, el número promedio de alelos por locus en el presente estudio fue mayor que los valores que se encontraron en $P$. resinosa $(A=9)$ y $P$. radiata $(A=$ 8.2) (Boys et al., 2005; Karhu et al., 2006).

Un número alto de alelos en $P$. patula en los huertos semilleros asexuales es importante debido a que las plantas que se produzcan a partir del germoplasma de estos huertos tienen mayor probabilidad de adaptarse a condiciones ambientales adversas que plantas que tienen origen en huertos semilleros donde el número de alelos es reducido; además, una diversidad genética alta en los huertos semilleros asexuales es necesaria para poder mantener individuos genéticamente sobresalientes en generaciones avanzadas de selección sin disminuir el potencial de adaptación de la especie (White et al., 2007); sin embargo, el número promedio de alelos por locus en la presente investigación fue menor al número promedio de A $=19.82$ que se reportó en $P$. oocarpa, una especie que tiene una distribución mayor, y de $A=11.36$ de $P$. tecunumanii (Dvorak et al., 2009). El número promedio de alelos por locus en la presente investigación fue inferior al número promedio de alelos por locus reportado por Rajora et al. 
(2000) y Chhatre y Rojora (2014) para P. strobus L. (A = 10.8 y $A$ = 9.4).

Los valores de heterocigosidad observada $(\mathrm{Ho})$ y heterocigosidad esperada (He) también mostraron un nivel alto de diversidad genética en los dos huertos semilleros estudiados. Estos valores fueron mayores que los encontrados en cinco poblaciones naturales de P. patula var. patula $(\mathrm{Ho}=0.475$ y $\mathrm{He}=0.567)$ y en una población de P. patula var. longipedenculata $(\mathrm{Ho}=0.548$ y $\mathrm{He}=0.599)$ (Dvorak et al., 2009). Los valores de Ho y He para P. patula también fueron mayores que los valores encontrados en $P$. resinosa $(\mathrm{Ho}=0.184$ y He $=0.508)$ (Boys et al., 2005) y $P$. strobus ( $\mathrm{Ho}=0.525$ y He $=0.608)$ (Chhatre y Rajora, 2014).

El valor de Ho para P. patula en el presente estudio fue mayor que el encontrado en $P$. oocarpa $(\mathrm{Ho}=0.545)$ y $P$. tecunumanii $(\mathrm{Ho}=549)$ (Dvorak et al., 2009); no obstante, $\mathrm{Ho}$ y $\mathrm{He}$ en los dos huertos semilleros fueron menores que los valores $\mathrm{Ho}=0.64$ y $\mathrm{He}=0.797$ reportados para poblaciones naturales de P. patula en la Sierra Juárez de Oaxaca (Alfonso-Corrado et al., 2014); además, los valores de He en P. oocarpa y P. tecunumanii fueron 0.711 y 0.646 , respectivamente (Dvorak et al., 2009). Karhu et al. (2006) reportaron una He de 0.73 para $P$. radiata y Thomas et al. (1999) una He de 0.74 para P. contorta Dougl. ex Loud. Las diferencias de heterocigosidad entre los estudios mencionados para P. patula y los resultados de la presente investigación pueden deberse al número de árboles incluidos en el estudio, ubicación geográfica, tamaño de las poblaciones y número de loci que se incluyeron en cada estudio.

En una población de producción, como son los huertos semilleros, es deseable tener valores bajos del índice de fijación para evitar efectos de depresión endogámica como consecuencia del cruzamiento entre individuos emparentados. El valor positivo del índice de fijación (F) indicó una deficiencia de heterocigotos en ambos huertos semilleros. El valor promedio de F para P. patula en ambos huertos fue inferior al valor $F=0.186$ que reportó para la misma especie Alfonso-Corrado et al. (2014). El valor de $F$ en el presente estudio también fue menor al valor que se encontró utilizando microsatélites en poblaciones de $P$. strobus ( $F=0.135)$ (Chhatre y Rajora, 2014) y Picea glauca (Moench) Voss ( $F=0.177$ ) (Fageria y Rajora, 2013).

El valor del índice de fijación total o índice de endogamia $\left(F_{I T}\right)$ fue bajo considerando ambos huertos semilleros. Los valores de $F_{I T}$ varían entre -1 y 1 ; valores positivos indica deficiencia de heterocigotos mientras que valores negativos significa un exceso de éstos (Hartl y Clark, 2007; Wright, 1965). En los huertos semilleros se esperaría un índice de fijación igual a cero debido a que los ortetos crecen a una distancia considerable, por lo que la probabilidad de algún progenitor común es baja. Los valores de $F_{1 T}$ para los loci NZPR5 y NZPR1078 expresaron los valores mayores de endogamia, mientras que el valor de $F_{\text {IT }}$ para el locus PtTX2123, con valor negativo, registró el menor nivel de apareamiento entre individuos emparentados. El índice de fijación $F_{\text {IT }}$ promedio en el presente estudio indicó una moderada deficiencia de individuos heterocigóticos en la población total (Wright, 1965). Deficiencias moderadas de heterocigotos también se encontraron en otras coníferas de polinización anemófila y dispersión de semilla por el viento como Picea glauca $\left(F_{1 T}=0.201\right)$ (Fageria y Rajora, 2013), Pinus sylvestris $L$. ( $F_{\text {IT }}=0.179$ para árboles tolerantes a contaminantes en el suelo y $F_{\mathrm{TT}}=0.057$ para árboles no tolerantes) (Chudzińska et al., 2016); asimismo, Boys et al. (2005) encontraron una deficiencia alta de heterocigotos $\left(F_{I T}=0.643\right)$ en $P$. resinosa. Las deficiencias de individuos heterocigotos se pueden deber a la endogamia, la selección en contra de heterocigotos o al efecto de Wahlund; es decir, una reducción en la heterocigosidad causada por la estructura de una subpoblación (Thomas et al., 1999).

El valor de $F_{\text {IS }}$ fue positivo tras considerar los siete loci, lo cual indicó una deficiencia de individuos heterocigóticos en ambos huertos semilleros de P. patula. El valor de $F_{\text {is }}$ para el locus NZPR1078 mostró el mayor valor de endogamia, mientras que el valor de $F_{\text {IS }}$ para el locus PtTX2123 expresó el valor menor de endogamia para ambos huertos semilleros asexuales. El valor del $F_{\text {IS }}$ para $P$. patula en el presente estudio fue menor a los valores promedio $\left(F_{\mid S}=0.146\right.$ y $\left.F=0.340\right)$ que Dvorak et al. (2009) y Alfonso-Corrado et al. (2014) encontraron para la misma especie. Otras coníferas como Picea glauca $\left(F_{1 S}=0.175\right)$ y Pinus strobus $\left(F_{I S}=0.139\right)$ (Chhatre y Rajora, 2014; Fageria y Rajora, 2013) presentaron valores mayores de $F_{\text {is }}$ que el obtenido para P. patula en la presente investigación. Para otras especies también se han encontrado valores altos de $F_{I S^{\prime}}$ como los valores reportados para $P$. contorta y $P$. resinosa con $F_{\text {IS }}=0.360$ y 0.504 , respectivamente (Boys et al., 2005; Thomas et al., 1999), mientras que en especies como P. brutia Ten. el valor de $F_{\text {IS }}$ fue bajo $\left(F_{1 S}=0.02\right)$ (Lise et al., 2007), lo que indicó una deficiencia mínima de heterocigotos dentro de las poblaciones.

El coeficiente de diferenciación $\left(F_{S T}\right)$ fue bajo entre huertos semilleros de $P$. patula, con base en el análisis de varianza molecular, con la mayoría de la diversidad genética entre individuos dentro de los huertos semilleros. La mayor parte de la diversidad genética dentro de poblaciones es común en la mayoría de las especies del género Pinus con amplia distribución (Ledig, 1998). El valor de $F_{S T}$ para P. patula en el presente estudio fue muy similar al valor $F_{S T}=0.025$ que se encontró en cinco poblaciones de esta especie (Dvorak et al., 2009), pero menor al de $F_{S T}=0.059$ que Alfonso-Corrado 
et al. (2014) encontraron para la misma especie. En P. contorta $\left(F_{S T}=0.028\right)$, P. brutia $\left(F_{S T}=0.07\right)$ y P. strobus $\left(F_{S T}=0.083\right)$ también se encontró diferenciación baja entre poblaciones, probablemente como consecuencia de un flujo alto de genes y tamaño grande entre sus poblaciones (Chhatre y Rajora, 2014; Lise et al., 2007; Thomas et al., 1999). Por otro lado, valores mayores $\left(F_{S T}=0.141\right.$ y $F_{S T}=$ 0.16 ) se encontraron en poblaciones de $P$. radiata (Karhu et al., 2006; Vogl et al., 2002). Bajo esta perspectiva, el nivel bajo de diferenciación genética que se encontró entre los huertos semilleros asexuales de P. patula en la presente investigación podría indicar un flujo alto de alelos en el pasado entre las poblaciones donde crecen los ortetos que originaron los rametos para establecer los huertos semilleros; ésto es común en poblaciones naturales de especies vegetales con polinización libre (Slatkin, 1981).

\section{CONCLUSIONES}

La diversidad genética de los huertos semilleros de Pinus patula fue alta, con deficiencia moderada de heterocigotos. El huerto semillero del ejido Villa Cuauhtémoc tuvo un nivel mayor de diversidad, aunque en La Selva se encontró un número mayor de alelos. La proporción mayor de la diversidad genética se ubicó dentro de los huertos, con una diferenciación genética baja entre éstos.

\section{AGRADECIMIENTOS}

Parte de la presente investigación se financió con presupuesto asignado a la Línea Prioritaria de Investigación 1 "Manejo Sustentable de Recursos Naturales" y del FIDEICOMISO No. 167304 de investigación del Colegio de Postgraduados. Los autores agradecen a los ejidatarios de los Ejidos La Selva en Huayacocotla, Veracruz y Villa Cuauhtémoc en Chignahuapan, Puebla, especialmente a los señores Juan Badillo y Luis Ordoñez Arroyo, presidentes del comisario ejidal, respectivamente, por las facilidades brindadas para la recolección del follaje.

\section{BIBLIOGRAFÍA}

Ahlinder J., T. J. Mullin and M. Yamashita (2014) Using semidefinite programming to optimize unequal deployment of genotypes to a clonal seed orchard. Tree Genetics and Genomes 10:27-34, https://doi.org/10.1007/s11295-013-0659-z

Alfonso-Corrado C., J. Campos-Contreras, G. Sánchez-García, A. Monsalvo-Reyes y R. Clark-Tapia (2014) Manejo forestal y diversidad genética de Pinus patula Schiede ex Schltdl. \& Cham. en Sierra Juárez, Oaxaca. Madera y Bosques 20:1122, https://doi.org/10.21829/myb.2014.202160

Allendorf F. W. and G. H. Luikart (2007) Conservation and the Genetics of Populations. Blackwell Publishing Ltd. Oxford, UK. 642 p.

Barrios D., V. García, L. F. Osorio, N. Isaza, J. D. Palacio, F. García y A. Sánchez (2004) Análisis de la diversidad genética de colecciones elite de Eucalyptus, por medio de marcadores moleculares microsatélites. Fitotecnia Colombiana 4:96-106.

Boys J., M. Cherry and S. Dayanandan (2005) Microsatellite analysis reveals genetically distinct populations of red pine (Pinus resinosa, Pinaceae). American Journal of Botany 92:833-841, https://doi.org/10.3732/ajb.92.5.833

Çengel B., Y. Tayanç, G. Kandemir, E. Velioglu, M. Alan and Z. Kaya (2012) Magnitude and efficiency of genetic diversity captured from seed stands of Pinus nigra (Arnold) subsp. pallasiana in established seed orchards and plantations. New Forests 43:303-317, https://doi.org/10.1007/s11056-011-9282-8

Chagné D., P. Chaumeil, A. Ramboer, C. Collada, A. Guevara, M. T. Cervera, G. G. Vendramin, V. Garcia, J. M. Frigerio, C. Echt, T. Richardson and C. Plomion (2004) Cross-species transferability and mapping of genomic and cDNA SSRs in pines. Theoretical and Applied Genetics 109:1204-1214, https://doi.org/10.1007/s00122-004-1683-z

Chhatre V. E. and O. P. Rajora (2014) Genetic divergence and signatures of natural selection in marginal populations of a keystone, long-lived conifer, eastern white pine (Pinus strobus) from Northern Ontario. PLOS ONE 9: e97291, https://doi.org/10.1371/journal.pone.0097291

Chudzińska E., K. Celiński, E. M. Pawlaczyk, A. Wojnicka-Półtorak and J. B. Diatta (2016) Trace element contamination differentiates the natural population of Scots pine: evidence from DNA microsatellites and needle morphology. Environmental Science and Pollution Research 23:22151-22162, https://doi.org/10.1007/s11356-016-7472-9

Devey M. E, J. C. Bell, T. L. Uren and G. F. Moran (2002) A set of microsatellite markers for fingerprinting and breeding applications in Pinus radiata. Genome 45:984-989, https://doi.org/10.1139/g02-064

Dvorak W. S., G. R. Hodge, J. E. Kietzka, F. Malan, L. F. Osorio and T. K. Stanger (2000) Pinus patula. In: Conservation and Testing of Tropical and Subtropical Forest Tree Species by the CAMCORE Cooperative. W. S. Dvorak, G. R. Hodge, J. L. Romero and W. W. Woodbridge (eds.). College of Natural Resources, North Carolina State University. Raleigh, North Carolina, USA. pp:149-173.

Dvorak W. S., K. M. Potter, V. D. Hipkins and G. R. Hodge (2009) Genetic diversity and gene exchange in Pinus oocarpa, a Mesoamerican pine with resistance to pitch canker fungus (Fusarium circinatum). International Journal of Plant Sciences 170:609626, https://doi.org/10.1086/597780

Elsik C. G., V. T. Minihan, S. E. Hall, A. M. Scarpa and C. G. Williams (2000) Low-copy microsatellite markers for Pinus taeda L. Genome 43:550-555, https://doi.org/10.1139/g00-002

Fageria M. S. and O. P. Rajora (2013) Effects of harvesting of increasing intensities on genetic diversity and population structure of white spruce. Evolutionary Applications 6:778-794, https://doi.org/10.1111/eva.12064

Feng F. J., X. Sui, M. M. Chen, D. Zhao, S. J. Han and M. H. Li (2010) Mode of pollen spread in clonal seed orchard of Pinus koraiensis. Journal of Biophysical Chemistry 1:33-39, https://doi.org/10.4236/jbpc.2010.11004

Fisher P. J., T. E. Richardson and R. C. Gardner (1998) Characteristics of single- and multi-copy microsatellites from Pinus radiata. Theoretical and Applied Genetics 96:969-979, https://doi.org/10.1007/s001220050828

Frankham R., J. D. Ballou and D. A. Briscoe (2004) A Primer of Conservation Genetics. Cambridge University Press. New York, USA. 220 p https://doi.org/10.1017/CB09780511817359

Fuentes-Amaro S. L., J. P. Legaria-Solano y C. Ramírez-Herrera (2019) Estructura genética de poblaciones de Pinus cembroides de la región central de México. Revista Fitotecnia Mexicana 42:5765, https://doi.org/10.35196/rfm.2019.1.57-65

Gaspar M. J., A. I. de-Lucas, R. Alía, J. A. P. Paiva, E. Hidalgo, J. Louzada, H. Almeida and S. C. González-Martínez (2009) Use of molecular markers for estimating breeding parameters: a case study in a Pinus pinaster Ait. progeny trial. Tree Genetics and Genomes 5:609-616, https://doi.org/10.1007/s11295-009-0213-1

Hartl D. L. and A. G. Clark (2007) Principles of Population Genetics. 4th edition. Sinauer Associates Inc. Publishers. Sunderland, Massachusetts, USA. 651 p.

Karhu A., C. Vogl, G. F. Moran, J. C. Bell and O. Savolainen (2006) Analysis of microsatellite variation in Pinus radiata reveals effects of genetic drift but no recent bottlenecks. Journal of Evolutionary Biology 19:167-175, https://doi.org/10.1111/j.1420-9101.2005.00982.x

Ledig F. T. (1998) Genetic variation in Pinus. In: Ecology and Biogeography of Pinus. D. M. Richardson (ed.). Cambridge University Press. New York, USA. pp:251-280. 
Lise Y., Z. Kaya, F. Isik, R. Sabuncu, I. Kandemir and S. Önde (2007) The impact of over-exploitation on the genetic structure of Turkish red pine (Pinus brutia Ten.) populations determined by RAPD markers. Silva Fennica 41:211-220, https://doi.org/10.14214/sf.291

Nei M. (1977) F-statistics and analysis of gene diversity in subdivided populations. Annals of Human Genetics 41:225233, https://doi.org/10.1111/j.1469-1809.1977.tb01918.x

Ortiz-Bibian M. A., D. Castellanos-Acuña, M. Gómez-Romero, R. LindigCisneros, M. Ángel Silva-Farías y C. Sáenz-Romero (2019) Variación entre poblaciones de Abies religiosa (H.B.K.) Schl. et Cham a lo largo de un gradiente altitudinal. I. Capacidad germinativa de la semilla. Revista Fitotecnia Mexicana 42:301-308, https://doi.org/10.35196/rfm.2019.3.301-308

Peakall R. and P. E. Smouse (2012) GenAlEx 6.5: genetic analysis in Excel. Population genetic software for teaching and research-an update. Bioinformatics 28:2537-2539, https://doi.org/10.1093/bioinformatics/bts460

Perry J. P. (1991) The Pines of Mexico and Central America. Timber Press. Portland, Oregon, USA. $231 \mathrm{p}$

Rajora O. P., M. H. Rahman, G. P. Buchert and B. P. Dancik (2000) Microsatellite DNA analysis of genetic effects of harvesting in old-growth eastern white pine (Pinus strobus) in Ontario, Canada. Molecular Ecology 9:339-348, https://doi.org/10.1046/j.1365-294x.2000.00886.x

Ramírez-Herrera C., J. J. Vargas-Hernández and J. López-Upton (1998) Allozyme variation in the Mexican closed-cone pines. In: Proceedings of an IFS Workshop. Recent Advances in Biotechnology for Tree Conservation and Management. S. Bruns, S. Mantell, C. Trägårdh and A. M. Viana (eds.), 15-19 September 1997. Florianópolis, Brazil. pp:89-93.

Sáenz-Romero C., H. Nienstaedt and J. J. Vargas-Hernández (1994) Performance of Pinus patula genotypes selected in South Africa and growing in their native Mexican environment. Silvae Genetica 43:73-81.

Slatkin M. (1981) Estimating levels of gene flow in natural populations.
Genetics 99:323-335

Stelzer H. E. and B. Goldfarb (1997) Implementing clonal forestry in the southeastern United States: SRIEG satellite workshop summary remarks. Canadian Journal of Forest Research 27:442-446, https://doi.org/10.1139/x96-200

Thomas B. R., S. E. Macdonald, M. Hicks, D. L. Adams and R. B. Hodgetts (1999) Effects of reforestation methods on genetic diversity of lodgepole pine: an assessment using microsatellite and randomly amplified polymorphic DNA markers. Theoretical and Applied Genetics 98:793-801, https://doi.org/10.1007/s001220051136

Valencia S., C. Ramírez y J. Vargas (1995) Comparación de diferentes métodos de selección en un ensayo de progenies de polinización libre de Pinus patula. In: Simposio sobre Avances en la Producción de Semillas Forestales en América Latina. R. Salazar (coord). Managua, Nicaragua. 16-20 octubre 1995 Centro Agronómico Tropical de Investigación y Enseñanza. Turrialba, Costa Rica. pp:63-69.

Vogl C., A. Karhu, G. Moran and O. Savolainen (2002) High resolution analysis of mating systems: inbreeding in natural populations of Pinus radiata. Journal of Evolutionary Biology 15:433-439, https://doi.org/10.1046/j.1420-9101.2002.00404.x

White T. L., W. T. Adams and D. B. Neale (2007) Forest Genetics. CABI Publishing. Cambridge, Massachusetts, USA. 682 p.

Wright S. (1965) The interpretation of population structure by F-statistics with special regard to systems of mating. Evolution 19:395-420, https://doi.org/10.2307/2406450

Wright S. (1969) Evolution and the Genetics of Populations. Volume 2. Theory of Gene Frequencies. University of Chicago Press. Chicago, Illinois, USA $511 \mathrm{p}$

Zelener N., S. N. Marcucci-Poltri, N. Bartoloni, C. R. López and H. E. Hopp (2005) Selection strategy for a seedling seed orchard design based on trait selection index and genomic analysis by molecular markers: a case study for Eucalyptus dunnii. Tree Physiology 25:1457-1467, https://doi.org/10.1093/treephys/25.11.1457 